\title{
Novel Self-Hybrid Mode-Locking of Semiconductor Lasers in an External Cavity
}

\author{
Bor-Lin Lee and Ching-Fuh Lin
}

\begin{abstract}
A new mode-locking method called self-hybrid modelocking is explored. This method uses RF modulation at the subharmonic of pulse repetition frequency so that both active and passive mode-locking mechanisms operate in the same gain region without the necessity of additional integrated absorbers. With the same cavity configuration, self-hybrid mode-locking can generate pulses about $2 \times$ shorter than the traditional active mode-locking.
\end{abstract}

Index Terms-Mode-locking, RF modulation, saturable absorption, superluminescent diode.

HORT-PULSE generation by mode-locking has attracted $\checkmark$ much attention for decades due to its wide applications in optical processing [1], medical and biological imaging [2], optical communication [3], [4], and so on. In particular, mode-locked semiconductor lasers are attractive due to their compactness and low cost. For semiconductor lasers, passive mode-locking methods usually generate shorter pulses than active mode-locking ones, but with worse timing jitter [5]. Hybrid mode-locking, combining active mode-locking and passive mode-locking, thus has both advantages of generating very short pulses with low timing jitter. However, it usually requires specially designed devices. The devices used for hybrid mode-locking typically have multiple sections or even multiple electrodes [6], [7]. Therefore, the hybrid mode-locking is relatively difficult and complex compared to other methods. In this letter, we report a novel self-hybrid mode-locking mechanism using external-cavity configuration. With suitable bias and RF modulation, this mechanism has the action of saturable absorption self-provided by the gain region of a conventional superluminescent diode (SLD), so multiple sections are no longer needed for the hybrid mode-locking.

The self-hybrid mode-locking is based on the cavity configuration of active mode-locking. Its operation principle is briefly described as follows. In active mode-locking, the RF modulation frequency is the same as the pulse repetition frequency, which is a multiple of the cavity round-trip frequency. As shown in Fig. 1(a), the pulses approximately coincide with the gain peaks of the RF modulation. In self-hybrid mode-locking, as demonstrated in Fig. 1(b), the RF modulation frequency is the half of the pulse repetition frequency. Therefore, the pulses pass through the gain media twice per modulation cycle: once at the gain peak and the other at the gain valley. In other words, as the

Manuscript received October 12, 1999; revised April 20, 2000. This work was supported in part by National Science Council, Taipei Taiwan, R.O.C., under Contract NSC 88-2112-M-002-038.

The authors are with the Graduate Institute of Electro-Optical Engineering and the Department of Electrical Engineering, National Taiwan University, Taipei, Taiwan, R.O.C. (e-mail: cflin@cc.ee.ntu.edu.tw)

Publisher Item Identifier S 1041-1135(00)07420-6. (a) active mode-locking
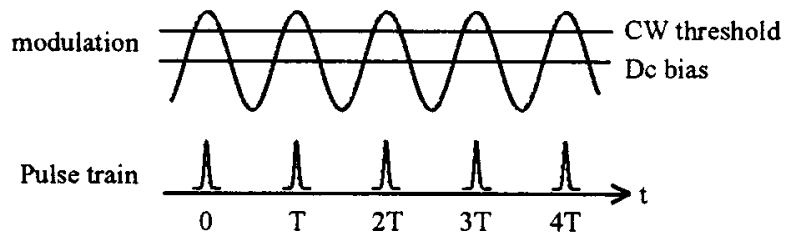

(b) self-hybrid mode-locking

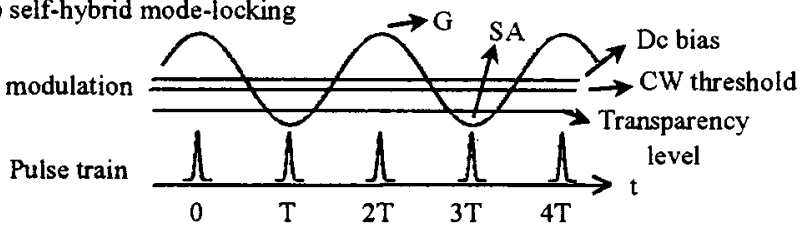

Fig. 1. Schematic of temporal behaviors in (a) active mode-locking and (b) self-hybrid mode-locking. (G: gain; SA: saturable absorber)

pulse leaves the SLD after the gain peak, it comes back to the SLD to see the gain valley next time. With suitable bias conditions, it is possible to make the carrier density at the gain valley lower than the transparency carrier density. The transparency carrier density is defined as the injected carrier density that makes light in the SLD experience neither gain nor loss. At this condition, the gain valley makes the SLD work like a saturable absorber. The gain peak provides the mechanism of active mode-locking, while the gain valley provides the effect of saturable absorption. With both effects, the self-hybrid mode-locking generates shorter pulses than traditional active mode-locking.

Subharmonic mode-locking has been demonstrated in monolithic configuration by RF modulation [8], [9] and in external cavity configuration by optical injection [10]. However, the RF modulation or the carrier modulation accompanied by optical injection used therein is usually very small, compared to the DC bias. In the self-hybrid mode-locking mechanism, the RF modulation is very deep so that the SLD is nearly reverse-biased and functions like a saturable absorber at the modulation valley.

To successfully achieve the self-hybrid mode-locking, the bias condition is very important. Fig. 2 schematically shows the typical gain-carrier density relation, where the transparency carrier density $\left(n_{t r}\right)$, the DC bias level $\left(n_{\mathrm{dc}}\right)$, and the amplitude of RF modulation in terms of carrier density $\left(n_{\bmod }\right)$ are indicated. Three criteria must be satisfied to have the self-hybrid mode-locking. First, the modulation frequency is the half of the pulse repetition frequency, as described previously. Second, $\left(n_{\mathrm{dc}}-n_{\mathrm{mod}}\right)<n_{t r}$, so the minimum gain $\left(G_{\min }\right)$ experienced by the pulse in each modulation cycle is lower than the transparency level to make the laser medium work like a saturable 
absorber. Third, the dc bias current must be higher than the cw threshold current. Because the gain increases sublinearly with the injected carrier density, as shown in Fig. 2, the increase of the gain at the gain peak, $\left|G_{\max }-G_{\mathrm{dc}}\right|$, is smaller than the decreased amount of the gain, $\left|G_{\mathrm{dc}}-G_{\min }\right|$, at the gain valley. Therefore, the DC bias has to be above the $\mathrm{cw}$ threshold in order that the pulses experience a positive net gain in each modulation cycle.

The cavity configuration for the experiment is shown in Fig. 3. The gain medium is a tilted-stripe SLD with the stripe tilted at $7^{\circ}$ to the facet normal in order to eliminate the facet reflectivity [11]. The device is $540 \mu \mathrm{m}$ long. The output light from the SLD was collimated by two collimators with $N A=0.55$. The linear cavity was formed with two arms: one using a grating aligned at the Littrow condition to feed back the light to the SLD, and the other using an output coupler with $R / T=50 / 50$. The Au-coated grating has 1200 lines $/ \mathrm{mm}$. Two lenses with $f=50 \mathrm{~mm}$ were used to focus the light onto the output coupler and to recollimate the output laser beam to increase the stability. The two arms have equal lengths, $l_{L}=l_{R}$. Both $c / 2 l_{L}$ and $c / 2 l_{R}$ are defined as the arm round-trip frequency, which is $803 \mathrm{MHz}$ in the experiment. Hence, the cavity round-trip frequency is $401.5 \mathrm{MHz}$. The lasing wavelength was tuned to $830 \mathrm{~nm}$ with the threshold current $50 \mathrm{~mA}$.

Active mode-locking was first tested in order to have a close comparison with the self-hybrid mode-locking. It should be noted that the fundamental RF frequency for active mode-locking in such a linear cavity with the gain in the center is $803 \mathrm{MHz}$ instead of $401.5 \mathrm{MHz}$. The device is biased below the threshold. The RF modulation is generated by the HP 83732B synthesized signal generator and amplified by the Mini-Circuits TIA 900-10 RF power amplifier (34 dB gain). The RF frequency near $803 \mathrm{MHz}$ was fine-tuned and the DC bias and the RF power were adjusted to optimize the active mode-locking. The optimal pulse is obtained at $10 \mathrm{~mA} \mathrm{DC}$ bias and $33 \mathrm{dBm}$ RF power. Because the device was not well impedance-matched, the RF power was not fully delivered to the SLD. The modulation current is estimated to be $48 \mathrm{~mA}$ at $33 \mathrm{dBm}$ RF power. A collinear autocorrelator was used for pulse-width measurement. The FWHM of autocorrelation trace is $26 \mathrm{ps}$ by active mode-locking.

After the active mode-locking experiment, the RF modulation frequency was reduced to $401.5 \mathrm{MHz}$ and the DC bias was increased to $66 \mathrm{~mA}$ to make self-hybrid mode-locking function. In order to turn the device into a saturable absorber at the modulation valley, the RF modulation has to be deep enough to assure that the device is biased below the transparency level. At the wavelength $830 \mathrm{~nm}$, the transparency current was measured to be about $36 \mathrm{~mA}$. Thus, the modulation current needs to be larger than $30 \mathrm{~mA}$. The corresponding RF power for the self-hybrid mode-locking effect is then about $30 \mathrm{dBm}$. In this situation, although the modulation frequency and the modulation depth are reduced, the pulses are still shortened due to the self-hybrid mode-locking.

Fig. 4 shows the autocorrelation width versus the modulation strength for self-hybrid mode-locking. Under shallow modulation, the effect of saturable-absorption is not significant. There-

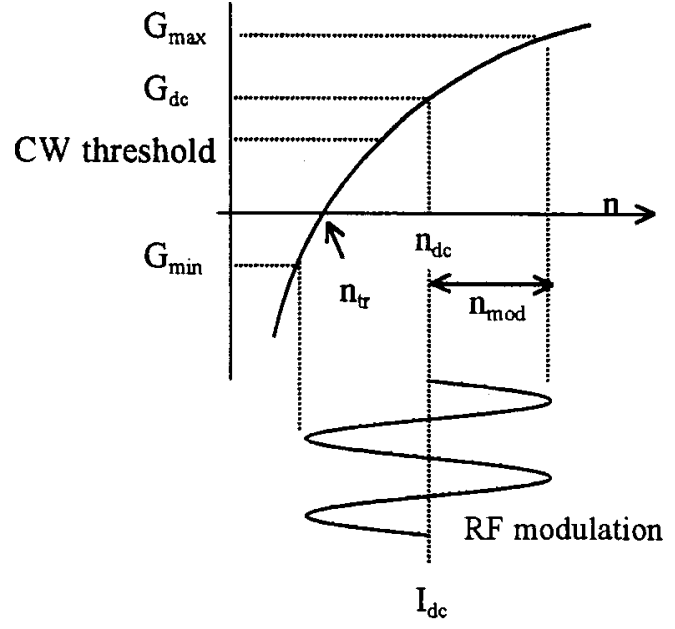

Fig. 2. Operation condition for self-hybrid mode-locking.

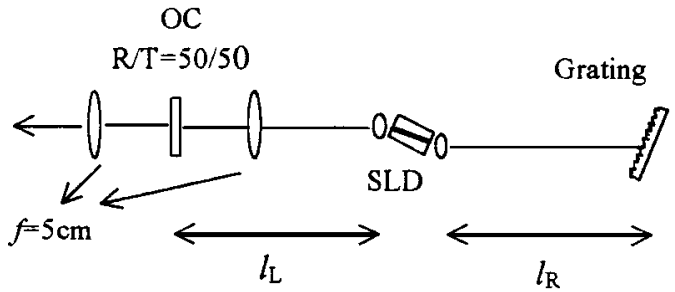

Fig. 3. The cavity configuration used for active mode-locking and self-hybrid mode-locking. (OC: output coupler)

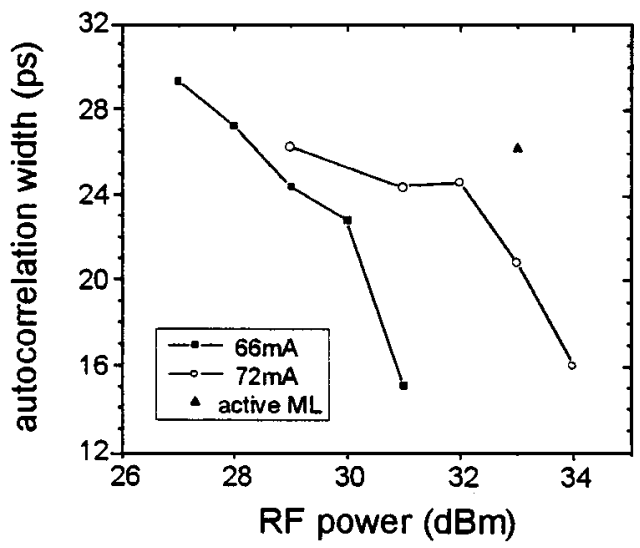

Fig. 4. Variation of the autocorrelation width with RF modulation power.

fore, the autocorrelation width slowly decreases to near $24 \mathrm{ps}$ when RF power increases to $\underline{30} \mathrm{dBm}$ for the $66 \mathrm{~mA}$ DC bias and $\underline{32} \mathrm{dBm}$ for the $72 \mathrm{~mA} \mathrm{DC}$ bias, respectively. The pulse width is still approximately equal to that from active modelocking. However, as the RF modulation is deep enough so that $n_{\text {mod }}>n_{\mathrm{dc}}-n_{t r}$, the saturable-absorption action rapidly reduces the pulse width. As shown in Fig. 4, the autocorrelation width rapidly decreases to $15 \mathrm{ps}$ and $16 \mathrm{ps}$ when RF power increases to $31 \mathrm{dBm}$ for the $66 \mathrm{~mA}$ DC bias and $34 \mathrm{dBm}$ for the 72 $\mathrm{mA}$ DC bias, respectively. Further increase of the RF modulation depth should lead to even shorter pulses. However, the gain medium at the modulation valley has a very strong absorption at the small injection current, as shown in Fig. 2. This results in a significant drop of the output power. 


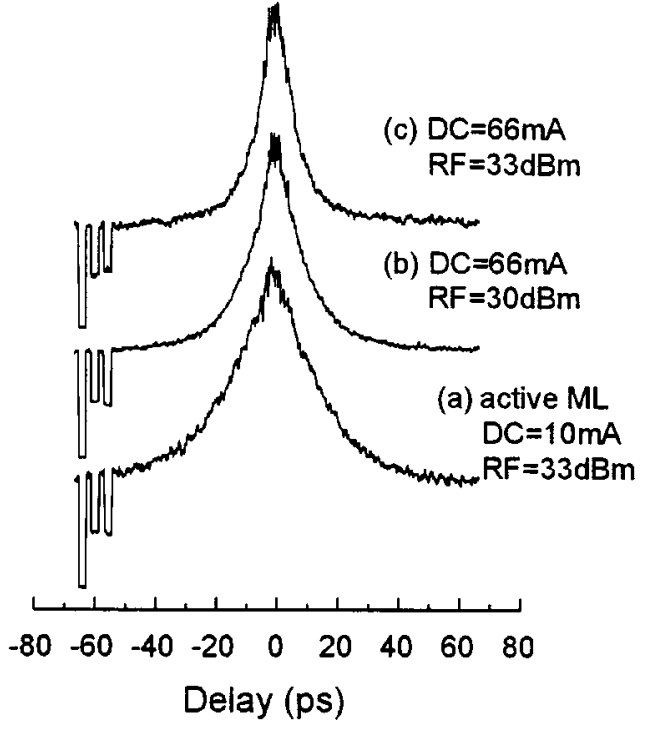

Fig. 5. Collinear autocorrelation traces: (a) active mode-locking; (b) and (c) self-hybrid mode-locking at different RF modulation power. The FWHM of autocorrelation trace for (a), (b), and (c) is $26 \mathrm{ps}, 16 \mathrm{ps}$, and $12 \mathrm{ps}$, respectively.

Two other cavity configurations had also been tested for the self-hybrid mode-locking and demonstrated similar behaviors. First, both arm lengths were reduced to $9.5 \mathrm{~cm}$, causing the modulation frequency to be $790 \mathrm{MHz}$ for self-hybrid mode-locking. At $68 \mathrm{~mA} \mathrm{DC}$ bias, the measured autocorrelation width could be as short as $14 \mathrm{ps}$ at $34 \mathrm{dBm} \mathrm{RF}$ power. Second, unequal arm lengths, $l_{L}=10 \mathrm{~cm}$ and $l_{R}=30 \mathrm{~cm}$, were used. The modulation frequency for self-hybrid mode-locking in this configuration is $750 \mathrm{MHz}$. The measured autocorrelation traces are shown in Fig. 5. For comparison, curve (a) shows the result obtained by active mode-locking in a similar cavity with the RF frequency $800 \mathrm{MHz}$. The autocorrelation width is $26 \mathrm{ps}$. Curves (b) and (c) show the results obtained by the self-hybrid mode-locking at $30 \mathrm{dBm}$ and $33 \mathrm{dBm}$ RF powers, respectively. The FWHM of the autocorrelation trace is 16 ps for Fig. 5(b) and 12 ps for Fig. 5(c). Again, the reduction of the pulse width by self-hybrid mode-locking is obvious.

The time-bandwidth products for the active mode-locking and the self-hybrid mode-locking are about 1.41 and 0.75 , respectively, by assuming hyperbolic-secant pulse shapes. The much smaller time-bandwidth product for self-hybrid modelocking indicates a reduced chirp. The reason may be due to the opposite sign of chirp contributed from the gain and the ab- sorption action, [12], [13]. They possibly compensate for one another to reduce the chirp.

In conclusion, we explore a new mode-locking technique that applies RF modulation at the subharmonic of the pulse repetition frequency. Both active and passive mode-locking mechanisms could then operate in the same gain region without the necessity of additional integrated absorbers. This method could shorten the pulses approximately to half of the actively modelocked ones. The width of the autocorrelation trace is about $12-16$ ps with a reduced time-bandwidth product.

\section{REFERENCES}

[1] A. J. Lowery and P. C. R. Gurney, "Comparison of optical processing techniques for optical microwave signal generation," IEEE Trans. Microwave Theory Tech., vol. 46, no. 2, pp. 142-150, 1998.

[2] R. R. Alfano, X. Liang, I. Wang, and P. P. Ho, "Time resolved imaging of translucent droplets in highly scattering turbid media," Science, vol. 264, pp. 1913-1915, 1994.

[3] M. R. H. Daza, H. F. Liu, M. Tsuchiya, Y. Ogawa, and T. Kamiya, "All-optical WDM-to-TDM conversion with total capacity of $33 \mathrm{~Gb} / \mathrm{s}$ for WDM network links," IEEE J. Quantum Electron., vol. 3, no. 5, pp. 1287-1294, 1997.

[4] R. Ludwig, A. Ehrhardt, W. Pieper, E. Jahn, N. Agrawal, H.-J. Ehrke, L. Kuller, and H. G. Weber, "40 Gbit/s demultiplexing experiment with $10 \mathrm{GHz}$ all-optical clock recovery using a modelocked semiconductor laser," Electron. Lett., vol. 32, no. 4, pp. 327-329, 1996.

[5] D. J. Derickson, P. A. Morton, J. E. Bowers, and R. L. Thornton, "Comparison of timing jitter in external and monolithic cavity mode-locked semiconductor lasers," Appl. Phys. Lett., vol. 59, no. 26, pp. 3372-3374, 1991.

[6] P. A. Morton, J. E. Bowers, J. A. Koszi, M. Soler, J. Lopata, and D. P. Wilt, "Monolithic hybrid mode-locked $1.3 \mu \mathrm{m}$ semiconductor lasers," Appl. Phys. Lett., vol. 56, no. 2, pp. 111-113, 1990.

[7] P. G. May and M. Bierbaum, "Monolithic mode-locking of long cavity GaAs-AlGaAs semiconductor lasers," Photon. Technol. Lett., vol. 3, no. 4, pp. 296-298, 1991.

[8] T. Hoshida, H.-F. Liu, M. Tsuchiya, Y. Ogawa, and T. Kamiya, "Subharmonic hybrid mode-locking of a monolithic semiconductor laser," IEEE J. Quantum Electron., vol. 2, no. 3, pp. 514-522, 1996.

[9] T. Hoshida, H. F. Liu, M. Tsuchiya, Y. Ogawa, and T. Kamiya, "Extremely low-amplitude modulation in a subharmonically hybrid modelocked monolithic semiconductor laser," IEEE Photon. Technol. Lett., vol. 8, no. 9, pp. 1160-1162, 1996.

[10] N. J. Traynor, H. F. Liu, and D. Novak, "Mode-locking of Fabry-Perot semiconductor laser by subharmonic optical injection," Electron. Lett., vol. 35 , no. 7 , pp. 566-567, 1999.

[11] W. Rideout, R. Holmstrom, J. Lacourse, E. Meland, and W. Powazinik, "Ultra-low-reflectivity semiconductor optical amplifiers without antireflection coatings," Electron. Lett., vol. 26, no. 1, pp. 36-38, 1990.

[12] M. Schell, M. Tsuchiya, and T. Kamiya, "Chirp and stability of modelocked semiconductor lasers," IEEE J. Quantum Electron., vol. 32, no. 7, pp. 1180-1190, 1996.

[13] A. Azouz, N. Stelmakh, and J.-M. Lourtioz, "Passive modelocking of semiconductor lasers with tunable group velocity dispersion cavity," Electron. Lett., vol. 29, no. 16, pp. 1437-1438, 1993. 